\title{
NOTES
}

\section{Novel Bimorph-Structured Hydrogel Containing Segregated Polymer Surfactant}

\author{
$\mathrm{Hu} \mathrm{YAN}^{1, \dagger}$ and Kaoru TsUJII ${ }^{1,2, \dagger}$ \\ ${ }^{1}$ Nanotechnology Research Center, Research Institute for Electronic Science, Hokkaido University, \\ Kita 10, Nishi 8, Kita-ku, Sapporo 001-0021, Japan \\ ${ }^{2} J S T$, CREST
}

(Received May 10, 2005; Accepted July 18, 2005; Published November 15, 2005)

\begin{abstract}
Bimorph-structured NIPA/NIPA-PMDP gel was fabricated from a NIPA gel containing a polymer surfactant (PMDP) by an electrophoretic method. The gel showed clearly bending motions when heating in $1 \mathrm{M} \mathrm{NaCl}$ solution. Directions of the bending motions can be changed by switching from pure water to the salt solution. It was verified that the NIPA-PMDP gel was more active material to the stimuli and NIPA gel was less active one in the bimorph structure, which resulted in the bending motions. [DOI 10.1295/polymj.37.858]

KEY WORDS Thermoresponsive Gel / Poly( $N$-isopropylacrylamide) (NIPA) / Polymer Surfactant

/ Volume Phase Transition / Bimorph Structure / Actuator /

[DOI 10.1295/polymj.37.858]
\end{abstract}

Stimuli-responsive hydrogels, which show dramatic volume change in response to change in environmental conditions such as temperature, $\mathrm{pH}$, concentration of salt, light, and electricity, have been extensively studied in fundamental and applied aspects. ${ }^{1-10}$ One of promising applications of hygrogels is soft actuators. Osada, et al. demonstrated the possibility of hydrogel actuator or artificial muscle in which electrical energy can convert into mechanical one. ${ }^{5}$ Bending motions are crucially important for the actuators. The bending motions are easily realized with bimorph structure in which one side (active material) changes in volume in response to stimuli and the other side (inactive material) does not as demonstrated with a conductive polymer. ${ }^{11} \mathrm{Hu}$, et al. ${ }^{12}$ first proposed and demonstrated a class of environmentally responsive materials based on the spatial modulation of the chemical nature of gels. They synthesized an internally heterogeneous or modulated structure by limiting the interpenetration of one gel network into another gel network. A bimorph-structured gel or bigel strip, one of their three simple applications, bends to a circle in response to a temperature increase or an increase in solvent concentration. ${ }^{12}$ The study demonstrated the clear concept of bimorph-structure of hydrogels for bending motions. However, few studies have been reported so far on other fabrication method of the bimorph-structured hydrogel probably because of the difficulty of adhesion of two kinds of hydrogels. In contrast, bimorph-structured actuators of conductive polymers have extensively been investigated ${ }^{13-16}$ after Baughman suggested a potential application of conductive polymers to artificial muscles and novel actuators. ${ }^{11}$ Jager et al. reported a possibility of single-cell manipulation by a microactuator prepared with polypyrrole. ${ }^{14}$ Recently $\mathrm{Lu}$ et al. have reported that $\pi$ conjugated polymer actuators have enhanced their lifetime up to 1 million cycles and the switching speeds of $100 \mathrm{~ms}$ for fast cycle. ${ }^{15}$ In the conductive polymer actuators the bimorph structures are easily fabricated because the conductive polymer films are real solid states. Even usual adhesion methods are applicable for the conductive polymer actuators.

Recently we have created $N$-isopropylacrylamid (NIPA, Figure 1a) gel system containing a polymer surfactant poly(2-(methacryloyloxyl)decylphosphate) (PMDP, Figure 1a). ${ }^{10}$ The gel system shows rapid volume change by stimulus of temperature as well as laser beam irradiation. Structurally the gel system contains the trapped micelles of PMDP inside the NIPA networks. An electrophoretic approach could make the gel system into two parts of the gel, i.e. NIPA-like and NIPA-PMDP-like gel parts (Figure 1b). ${ }^{10}$ Based on our previous studies, we suggest a strategy to create bimorph structures of hydrogels and verify it. Herein we report a novel and convenient fabrication of bimorph-structured hydrogel system which would provide a guiding principle for fabrication of bimorph-structured actuators.

\footnotetext{
${ }^{\dagger}$ To whom correspondence should be addressed (Tel: +81-11-706-9348 or 9356, FAX: +81-11-706-9357, E-mail: yanhu@es.hokudai.ac.jp or tsujik@es.hokudai.ac.jp).
} 
a)

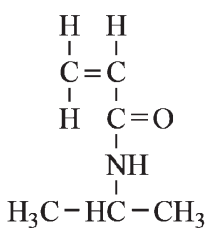

N-Isopropylacrylamide (NIPA)

b)

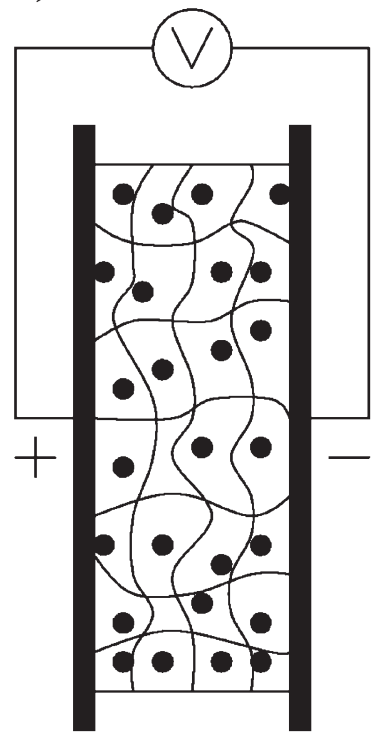

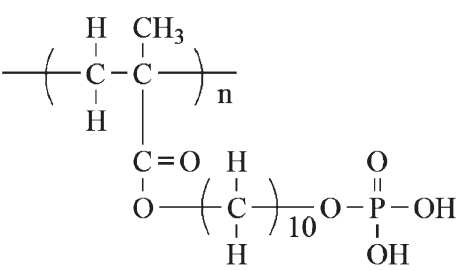

Poly(2-(Methacryloyloxyl)decylphosphate) (PMDP)

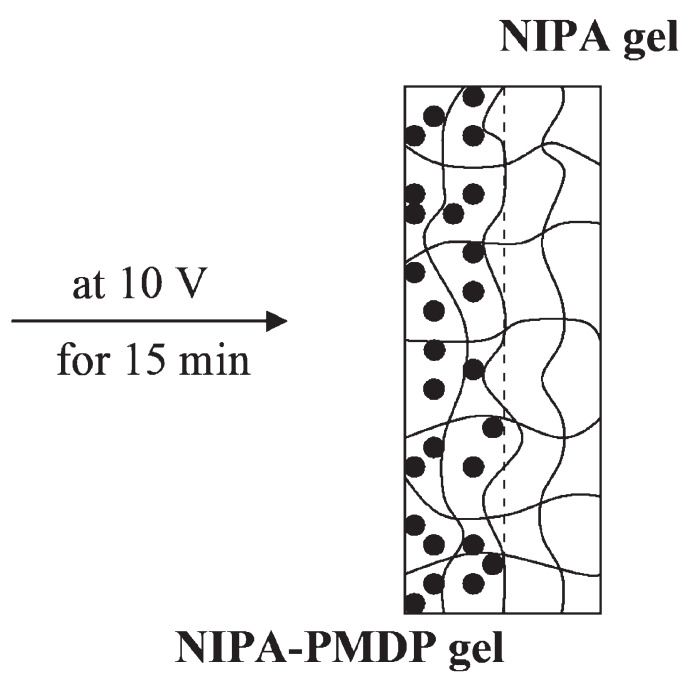

Figure 1. a) Chemical structures of gel monomer (NIPA) and polymer surfactant (PMDP). b) Fabrication method of bimorphstructured hydrogel. Solid lines are polymer networks of NIPA gel and black dots are PMDP micelles.

\section{EXPERIMENTAL}

\section{Materials}

$N$-isopropylacrylmide (NIPA), $N, N^{\prime}$-methylenebisacrylamide (MBA), ammonium persulphate (APS), tetramethylethylenediamine (TEMED) were used as received from Wako Pure Chemical Industries, Ltd. Poly(2-(methacryloyloxyl)decylphosphate) (PMDP) was synthesized from 2-(methacryloyloxyl)decylphosphate (MDP) which was synthesized according to a previous report. ${ }^{17}$ An oil-soluble dye, Yellow $\mathrm{AB}$, was obtained from Tokyo Kasei Kogyo Co., Ltd.

\section{Preparation of Bimorph-structured Gels}

The bimorph-structure of the hydrogels was prepared as follows: NIPA-PMDP gel was synthesized in a capillary $(2.0 \mathrm{~mm}$ in diameter) and withdrawn from the capillary according to procedure previously reported by us. ${ }^{10}$ The NIPA-PMDP gel was washed with pure water by immersing for $24 \mathrm{~h}$. Two sides of the cylindrical gel were contacted with two electrodes, and electric voltage of $10 \mathrm{~V}$ was applied for $15 \mathrm{~min}$ along the width of the cylindrical gel, as shown in Figure 1b. Dye-solubilization experiments were carried out to confirm migration of the PMDP. Yellow $\mathrm{AB}$ was used as an oil-soluble dye.

For comparison, the bimorph-structure of hydrogels was fabricated by two-step synthesis. In the first step, NIPA gel was synthesized in a capillary $(1.3 \mathrm{~mm}$ in diameter). ${ }^{10}$ The cylindrical NIPA gel was withdrawn from the capillary, and inserted into another capillary ( $2.0 \mathrm{~mm}$ in diameter). NIPA-PMDP gel was synthesized in the NIPA gel-inserted capillary as the second step. Finally the bimorph NIPA/NIPA-PMDP gel actuator was withdrawn, and extensively washed with pure water by immersing. Observation of the bending motion of the bimorph-structured hydrogel was carried out by heating/cooling or switching medium from pure water into salt solution. The typical observation was as follows: The actuating properties of the bimorph NIPA/NIPA-PMDP gels were observed in a beaker when heating or cooling by temperaturecontrollable thermoelectric device. The bending of the gel in pure water or in $1 \mathrm{M} \mathrm{NaCl}$ solution was recorded by a digital camera. 


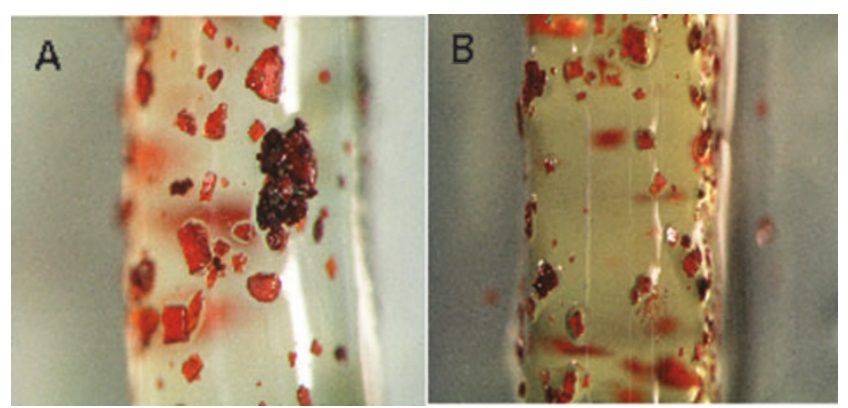

Figure 2. NIPA-PMDP gels after (A) and before (B) electrophoretic treatment.

\section{RESULTS AND DISCUSSION}

The molecules of the polymer surfactant, PMDP, were moved to the anodic side by an electrophoretic manner. Thus, the bimorph NIPA/NIPA-PMDP gels were prepared. To confirm the bimorph-structure dyesolubility experiments were carried out for the cylindrical gels before and after the electrophoretic treatment. In this experiment Yellow AB was chosen as an oil-soluble dye which is insoluble in pure water but soluble in the hydrophobic moiety of surfactant micelles. We reported previously the micelle formation of the PMDP in the NIPA-PMDP gel. ${ }^{10}$ Figure 2 shows microscopic images of the NIPA-PMDP gels containing the solubilized dye molecules before and after the electrophoretic treatment. The gel before the treatment showed entirely yellowish color, indicating that entire domain of the gel contains PMDP micelles, as shown in Figure 2B. On the other hand, the gel after the treatment, however, showed yellowish color in one side, but not in the other side (Figure $2 \mathrm{~A})$. It is indicated that only the yellow-colored side contains the PMDP micelles. Adsorption of the Yellow $\mathrm{AB}$ powder is also consistent with the consideration. The side containing PMDP micelles should be more hydrophobic, thus more Yellow $\mathrm{AB}$ powder adheres on this side. The experimental results visually confirmed the bimorph-structure of the gel in which one side is NIPA-PMDP-like, another side is NIPAlike gel.

It is considerable that the polymeric surfactant PMDP micelles, even unimers hardly migrate through the NIPA gel networks without the electric field. Therefore no release of the PMDP was observed even after repeated volume changes of the NIPA-PMDP. ${ }^{10}$ The PMDP micelles and unimers should be dynamically equilibrated. ${ }^{18}$ It is easily imaged that the PMDP unimers could migrate through the gel networks under a sufficient electric field, since the size of the unimers is similar to proteins which migrate easily in conventional electrophoresis. ${ }^{19}$ The segregated PMDP mole-

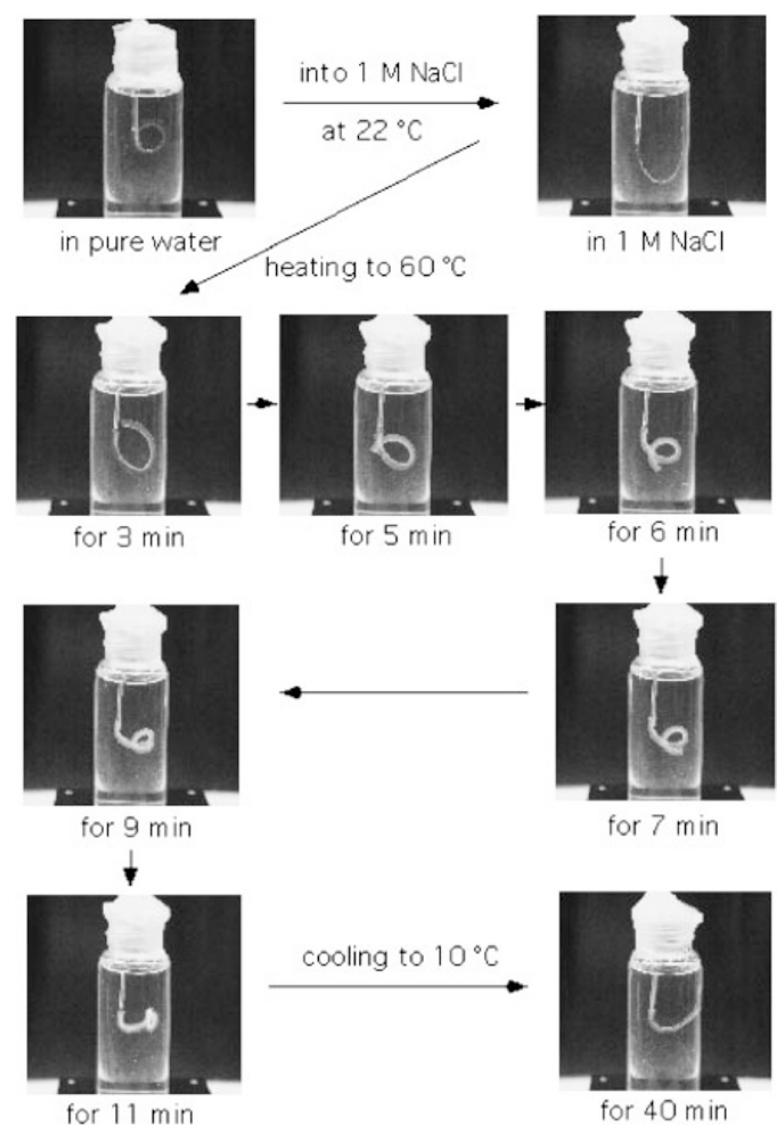

Figure 3. Bending motions of the bimorph-structured hydrogel.

cules inside the NIPA gel do not diffuse back because of the entanglement of the polymer chains with the gel networks. ${ }^{10}$

Figure 3 showed bending motions of the bimorphstructured NIPA/NIPA-PMDP gel. Initial shape of the gel after the electrophoretic treatment was coiled depending on the experimental conditions of electrophoresis. The gel stretched partly first after the medium changed from pure water into $1 \mathrm{M} \mathrm{NaCl}$ solution because the NIPA gel shrunk more rapidly than the NIPA-PMDP gel when the charged ions penetrate into the gel network. The polymer surfactant PMDP is ionized in the gel network, therefore an electrostatic shield influences much more in the NIPA-PMDP gel than in the NIPA gel which is non-ionic network. The gel would bend along opposite direction due to the same reason when the medium changes from salt solution to pure water. The partly stretched gel started to bend further when it was heated to $60^{\circ} \mathrm{C}$ above the phase transition temperature of NIPA gel $\left(\sim 34^{\circ} \mathrm{C}\right)$. The NIPA-PMDP gel shrinks much more rapidly than the NIPA gel in both pure water and salt solution above the phase transition temperature. ${ }^{10}$ The gel continued the bending motion with increasing heating time to result in a multi-coiled shape. The coiled gel can be stretched again to the initial shape after cooling 
to $10^{\circ} \mathrm{C}$ for $40 \mathrm{~min}$. It is noteworthy that the bimorphstructured NIPA/NIPA-PMDP gel could change the bending direction when medium changes from pure water to salt solution, or from salt solution to pure water at constant temperature.

In the pure water the bimorph-structured NIPA/ NIPA-PMDP gel showed similar bending motions when heating. In the gel NIPA-PMDP part should be more active side and NIPA part should be less active one. To verify it we fabricated similar bimorphstructure by two-stepped synthesis. In this fabricated gel one side is exactly NIPA-PMDP gel and the other side is exactly NIPA gel. This bimorph-structured NIPA/NIPA-PMDP gel showed similar bending motions both in pure water and $1 \mathrm{M} \mathrm{NaCl}$ solution when heating above the phase transition temperature.

\section{CONCLUSIONS}

We have succeeded in fabricating the bimorphstructured NIPA/NIPA-PMDP gel by an electrophoretic method. The gel showed clearly bending motions when heating in $1 \mathrm{M} \mathrm{NaCl}$ solution. Directions of the bending motions are changed by switching pure water to the salt solution. In the bimorph structure the NIPA-PMDP gel was more active material and NIPA gel was less active one, which resulted in the bending motions. These experimental results could provide a guiding principal for realizing feasible soft actuators.

Acknowledgment. H.Y. acknowledges fellowship support from 21st century COE program "Center of Excellence for Advanced Life Science on the Base of Bioscience and Nanotechnology" of Hokkaido University, Japan.

\section{REFERENCES}

1. T. Tanaka, Phys. Rev. Lett., 40, 820 (1978).

2. Y. Hirokawa and T. Tanaka, J. Chem. Phys., 81, 6379 (1984).

3. A. Suzuki and T. Tanaka, Nature, 346, 345 (1990).

4. Y. Zhang, T. Tanaka, and M. Shibayama, Nature, 360, 142 (1992).

5. Y. Osada, H. Okuzaki, and H. Hori, Nature, 355, 242 (1992).

6. R. Yoshida, K. Uchida, Y. Kaneko, K. Sakai, A. Kikuchi, Y. Sakurai, and T. Okano, Nature, 374, 240 (1995).

7. C. Wang, R. J. Stewart, and J. Kopecek, Nature, 397, 417 (1999).

8. Y. Murase, K. Tsujii, and T. Tanaka, Langmuir, 16, 6385 (2000).

9. J. A. Hinkley, L. D. Morgret, and S. H. Gehrke, Polymer, 45, 8837 (2004).

10. H. Yan, H. Fujiwara, K. Sasaki, and K. Tsujii, Angew. Chem. Int. Ed., 44, 1951 (2005).

11. R. H. Baughman, Makromol. Chem. Macromol. Symp., 51, 193 (1991).

12. Z. Hu, X. Zhang, and Y. Li, Science, 269, 525 (1995).

13. Q. Pei and O. Inganas, Synth Met., 55-57, 3718 (1993).

14. E. W. H. Jager, O. Inganas, and I. Lundstrom, Science, 288, 2335 (2000).

15. W. Lu, A. G. Fadeev, B. Qi, E. Smela, B. R. Mattes, J. Ding, G. M. Spinks, J. Mazurkiewicz, D. Zhou, G. G. Wallace, D. R. MacFarlane, S. A. Forsyth, and M. Forsyth, Science, 297, 983 (2002).

16. H. Yan, K. Tomizawa, H. Ohno, and N. Toshima, Macromol. Mater. Eng., 288, 578 (2003).

17. T. Koudate and Y. Nakano, JP Patent P2000-26481A, 2002.

18. B. Jonsson, B. Lindman, K. Holmberg, and B. Kronberg, "Surfactants and Polymers in Aqueous Solution," John Wiley \& Sons, New York, 1998.

19. B. Alberts, D. Bray, J. Lewis, M. Raff, K. Roberts, and J. D. Watson, "Molecular Biology of the Cell," Garland Publishing, Inc., New York, 1989. 